\title{
miRNA-186 inhibits prostate cancer cell proliferation and tumor growth by targeting YY1 and CDK6
}

\author{
SHU LU, MING-SHAN WANG, PEI-JIE CHEN, QIANG REN and PEIMING BAI \\ Department of Urology, Zhongshan Hospital Affiliated to Xiamen University, Xiamen, Fujian 361004, P.R. China
}

Received February 22, 2016; Accepted February 24, 2017

DOI: 10.3892/etm.2017.4387

\begin{abstract}
RNAs (miRNAs) are known to be important in tumor initiation and progression. Recent studies have demonstrated that miR-186 is critical in several types of cancer, including human non-small cell lung cancer, bladder cancer and pancreatic ductal adenocarcinoma. However, the functions of miR-186 in prostate cancer (PCa) are still unclear. In the present study, downregulation of miR-186 in PCa cells was detected when compared with the normal prostate cell line. When miR-186 overexpressed in PCa cells, cell proliferation in vitro was evidently inhibited as shown using cell counting kit-8 assays and cell-cycle analysis, and tumor growth in vivo was decreased as shown by tumor growth assays in nude mice. Furthermore, through bioinformatics prediction and biochemical analyses, Yin Yang 1 (YY1) and cyclin-dependent kinase 6 (CDK6) have been proven to act as direct targets of miR-186. These results indicate that miR-186 is a negative regulator in $\mathrm{PCa}$ by inhibiting PCa cell proliferation via targeting YY1 and CDK6.
\end{abstract}

\section{Introduction}

Prostate cancer ( $\mathrm{PCa}$ ) is the most common type of cancer detected among males in the USA, and the morbidity has evidently increased in the past decade in China (1). Several different strategies are currently being used for PCa therapy, of which androgen-deprivation therapy (ADT) is the most common. However, many PCa patients eventually become resistant to ADT and develop castration-resistant prostate cancer (CRPC), which is more difficult to treat $(2,3)$. Unfortunately, the mechanisms underlying the development of PCa and CRPC are not clearly understood. Therefore, the lack of understanding regarding the molecular mechanisms underlying PCa emphasizes the need to identify new molecular

Correspondence to: Dr Peiming Bai, Department of Urology, Zhongshan Hospital Affiliated to Xiamen University, 209 Hubin South Road, Xiamen, Fujian 361004, P.R. China

E-mail: baipeiming@xmu.edu.cn

Key words: Yin Yang 1, cyclin-dependent kinase 6, microRNA, miR-186, prostate cancer, cell proliferation, tumor growth mechanisms, which may help develop new strategies for $\mathrm{PCa}$ diagnosis, treatment and prognosis.

Mature microRNAs (miRNAs) are small (18-22 bp) nucleotides and non-coding RNAs that can regulate the translation and degradation of mRNAs by binding to the 3'-untranslated regions (3'-UTRs) of the target mRNAs $(4,5)$. miRNAs are known to be critical during carcinogenesis by regulating cell proliferation, apoptosis and tumor metastasis $(6,7)$. Furthermore, several studies have demonstrated that miRNAs such as miR-29s, miR-155 and miR-181 may act as regulators and biomarkers in PCa (8-10). Furthermore, miR-186 has been shown to be downregulated in PCa specimens and was inversely correlated with clinicopathological parameters (11). However, the molecular mechanism of miR-186 in the progress of PCa is largely unknown.

The present study demonstrated that miR-186 is downregulated in PCa cells. miR-186 overexpression inhibits PCa cell proliferation in vitro and suppresses tumor growth of PCa cells in vivo. Additionally, miR-186 was capable of downregulating the expression of Yin Yang 1 (YY1) and cyclin-dependent kinase 6 (CDK6) by binding to their 3'-UTRs. Finally, the present study demonstrated that miR-186 has a tumor suppressive role in the PCa progress.

\section{Materials and methods}

Cell lines. The adult human prostatic epithelial cell line RWPE-1 and human PCa lines PC-3, DU-145 and LNCAP were all purchased from the Chinese Academy of Sciences (Shanghai, China). The RWPE-1 cell lines were cultured in keratinocyte-serum-free medium (Invitrogen; Thermo Fisher Scientific, Inc., Waltham, MA, USA) supplemented with $25 \mathrm{mg}$ bovine pituitary extract (13028-014) and $2.5 \mu \mathrm{g}$ recombinant human epidermal growth factor (10450-013; both Invitrogen; Thermo Fisher Scientific, Inc.). Other PCa lines were all cultured in RPMI 1640 medium (Invitrogen; Thermo Fisher Scientific, Inc.), supplemented with $10 \%$ fetal bovine serum, $100 \mathrm{IU} / \mathrm{ml}$ penicillin and $100 \mathrm{mg} / \mathrm{ml}$ streptomycin (Invitrogen; Thermo Fisher Scientific, Inc.).

Plasmid construction and generation of stable cell lines. The sequence of the miR-186-containing flank region was amplified from human genomic DNA using polymerase chain reaction (PCR) and inserted into pCDH-CMV-EF1-GFP+puro (System Biosciences, Mountain View, CA, USA). In order 
to generate an miR-186-expressing stable cell line, a lentivirus-mediated packaging system containing four plasmids, pCDH-miR-186 or control plasmid, pMDL, REV and VSVG, at the ratio (quantity) of 5:5:2:3, was created. The transfection and lentiviral infection processes were performed according to those previously described (12). In order to downregulate miR-186 in the PC-3 cells, an miR-186 inhibitor synthesized by Guangzhou RiboBio Co., Ltd. (Guangzhou, China) was used according to the manufacturer's instructions.

For the construction of human CDK6, YY1, vascular endothelial growth factor A (VEGFA) and mitogen-activated protein kinase kinase kinase 2 (MAP3K2) 3'-UTR wild-type and mutant region luciferase reporters, the indicated 3 '-UTR regions were amplified from HEK293T cDNA using PCR amplification with the primerstar polymerase (Takara Biotechnology Co., Ltd., Dalian, China) and the appropriate primers (see List of primers used for the construction of luciferase reporters). The resultant PCR fragments were cloned into the pMIR-report vector (Promega Corporation, Madison, WI, USA) using the SpeI and HindIII sites.

hCDK6 3'-UTR forward, AAGCTGCGCACTAGTCAT TCAAGGTCAGGTCTACT and reverse, TCCTTTATTAAG CTTACAACTACACCGATGGAAG; hCDK6 3'-UTR-Mut forward, GAATTTTTCAGCATTGGGGAGAATTTTAAA TGAGTAGAG and reverse, CTCATTTAAAATTCTCCCC AATGCTGAAAAATTCCAGTT; hYY1 3'-UTR forward, AAGCTGCGCACTAGTGCATCTTCCAGAAGTGTGA and reverse, TCCTTTATTAAGCTTCGTAGCATTACTAAG CATATCC; hYY1 3'-UTR-Mut forward, CATCAAAAGACA ATTGGGGATACAACAGTGCTAAAAATG and reverse, TTAGCACTGTTGTATCCCCAATTGTCTTTTGATGCG AAG; hMAP3K2 3'-UTR forward, AAGCTGCGCACTAGT CCTCTATCACTGACACATCA and reverse, TCCTTTATT AAGCTTGCAGAAAGCTATCCCTCAA; and hVEGFA 3'-UTR forward, TAAAATATATATATTGGGGTTTTAAAT TAACAGTGCTAA and reverse, ACTGTTAATTTAAAA CCCCAATATATATATTTTATATAT.

Quantitative PCR (qPCR) and QuickChange PCR. Total RNA was obtained using TRIzol reagent (Invitrogen; Thermo Fisher Scientific, Inc.) and the enriched miRNA molecules were prepared with an E.Z.N.A. ${ }^{\circledR}$ miRNA kit (Omega Bio-Tek, Inc., Norcross, GA, USA) according to the manufacturer's instructions. For miRNA reverse transcription, cDNA was obtained using a Thermo Scientific ${ }^{\circledR}$ RevertAid $^{\circledR}$ First Strand cDNA Synthesis kit (Thermo Fisher Scientific, Inc.) with 100 ng total mRNA. Furthermore, mRNA was reverse transcribed using an RT-PCR kit (Invitrogen; Thermo Fisher Scientific, Inc.) according to the manufacturer's instructions. qPCR was performed with primers for miR-186, CDK6, MAP3K2, nuclear receptor coactivator 2 (NCOA2), VEGFA, PMEPA, RB binding protein 9 (RBBP9), X-linked inhibitor of apoptosis protein (XIAP), transforming growth factor $\beta$ receptor 2 (TGF $\beta$ R2), YY1 and folate hydrolase 1 (FOLH1) using SYBR Green PCR Master Mix (Invitrogen; Thermo Fisher Scientific, Inc.) in a real-time PCR System (Applied Biosystems; Thermo Fisher Scientific, Inc.) following a standard qPCR procedure, as follows: Denaturation at $95^{\circ} \mathrm{C}$ for $5 \mathrm{~min}$; followed by 38 cycles of $95^{\circ} \mathrm{C}$ for $30 \mathrm{sec}, 58^{\circ} \mathrm{C}$ for $30 \mathrm{sec}$ and $72^{\circ} \mathrm{C}$ for $20 \mathrm{sec}$; and a final step of $72^{\circ} \mathrm{C}$ for $7 \mathrm{~min}$.
The primer sequences used were the following: miR-186, 5'-GCGGCGGCAAAGAATTCTCC-3' and 3'-ATCCAG TGCAGGGTCCGAGG-5'; CDK6, 5'-CACCCCAACGTG GTCAGGTT-3' and 3'-TTCAGTGGGCACTCCAGGCT-5'; MAP3K2, 5'-AACAGAATGATGTCCGAG-3' and 3'-ATA GACTGTCCAAAGGCA-5'; NCOA2, 5'-AGAGACAAG AAAGCGCAA-3' and 3'-TTCCTGTTCACGATTACG-5'; VEGFA, 5'-CACGGTCCCTCTTGGAAT-3' and 3'-ATG TGGGTGGGTGTGTCT-5'; PMEPA, 5'-TCGGAGAGCACA GTGTCA-3' and 3'-TGCAGGTACGGATAGGTG-5'; RBBP9, 5'-CTGCCCTTCATGGAGACA-3' and 3'-TCCCCCAAG TCTGATGTG-5'; XIAP, 5'-AGGAGCAGCTTGCAAGAG-3' and 3'-GTCTTCACTGGGCTTCCA-5'; TGFBR2, 5'-TGT GATGTGAGATTTTCCAC-3' and 3'-GCAAACTGTCTC TAGTGTTATG-5'; YY1,5'-CAACAAGAAGTGGGAGCA-3' and 3'-CCACTGTCTCATGGTCAA-5' and FOLH1, 5'-TTC TATGAAACATCCACAGG-3' and 3'-GTTGCTTTTGTC AAAGTCCT-5'. The relative quantification was performed by normalizing against the levels of glyceraldehyde 3-phosphate dehydrogenase for mRNA or U6 for miRNA. For mutation type of CDK6 and YY1, QuickChange PCR was performed, as follows: Denaturation at $94^{\circ} \mathrm{C}$ for $3 \mathrm{~min}$; followed by 16 cycles of $94^{\circ} \mathrm{C}$ for $30 \mathrm{sec}, 56-60^{\circ} \mathrm{C}$ for $2 \mathrm{~min}$ and $68^{\circ} \mathrm{C}$ for $10 \mathrm{~min}$; and a final step of $68^{\circ} \mathrm{C}$ for $30 \mathrm{~min}$.

Luciferase-reporter activity assay. HEK293T, purchased from the Chinese Academy of Sciences (Shanghai, China), or PC-3 cells were seeded into a 24-well plate and co-transfected with miR-186 or control and 3'-UTR-luciferase plasmids. The cells were then lysed $48 \mathrm{~h}$ post-transfection, and the luciferase activity was measured using a Dual-Glo Luciferase Assay System (Promega Corporation, Madison, WI, USA) and normalized against renilla luciferase activity. Each treatment was performed in triplicate in three independent experiments.

Western blot analysis. HEK293T and PC-3 cells were lysed, proteins were extracted and western blotting was performed, as previously described (13). Equal amounts of cell lysates $(15 \mu \mathrm{g})$ were fractionated by size on a $10 \%$ SDS-polyacrylamide gel (Beijing Solarbio Science \& Technology Co., Ltd., Beijing, China) and transferred onto polyvinylidene fluoride membranes (EMD Millipore, Billerica, MA, USA). The membrane was blocked with tris-buffered saline-Tween with $5 \%$ non-fat milk at room temperature for $1 \mathrm{~h}$. Samples were then incubated with antibodies against the following proteins: YY1 (CST-2185S; 1:2,000; Cell Signaling Technology, Inc., Danvers, MA, USA), CDK6 (D155263; 1:1,000; Sangon Biotech Co., Ltd., Shanghai, China) and $\beta$-actin (MABT825; 1:10,000; EMD Millipore) at $4^{\circ} \mathrm{C}$ overnight. Subsequently, horseradish peroxidase-labeled goat anti-rabbit (ab6721; 1:10,000; Abcam, Cambridge, MA, USA) and goat anti-mouse (ab6789; 1:10,000; Abcam) secondary polyclonal antibodies were added, respectively, followed by immobilon enhanced chemiluminescence (EMD Millipore).

Cell cycle assays. For cell cycle analysis, PC-3 cells were harvested $72 \mathrm{~h}$ after transfection, and then measured using a cell cycle staining kit (Multi Sciences Biotech Co., Ltd., Hangzhou, China) according to the manufacturer's instructions and using flow cytometry. The percentage of cells in the 
G0/G1, S and G2/M phases of the cell cycle are presented in the results. All experiments were performed in triplicate and repeated three times with similar results.

Cell proliferation assay. For the cell proliferation assay, the cell suspension $(2,000$ cells/well) was inoculated in a 96-well plate. After 2 or 3 days of transfection, $10 \mu \mathrm{l}$ cell counting kit-8 (CCK8) solution (Dojindo Molecular Technologies, Inc., Kumamoto, Japan) was added into each well. Next, the cell suspension was incubated for $4 \mathrm{~h}$ and the absorbance was measured at $450 \mathrm{~nm}$ using a microplate reader.

Tumor formation assays in a nude mouse model. All experimental protocols were approved and the methods were performed in accordance with the Guidelines of the Animal Care and Use Committee of Xiamen University (Xiamen, China). A total of 8 specific-pathogen-free (SPF) mice were obtained from Xiamen University Laboratory Animal Center (Xiamen, China). All SPF mice were housed in a standard animal facility under standard conditions (temperature, $20-23^{\circ} \mathrm{C}$; humidity, $55 \pm 5 \%$; 12 -h light/dark cycle) and had access to the same normal diet and water ad libitum, meeting the SPF system required. For the tumor growth assay, $1 \times 10^{6} \mathrm{PC}-3 / \mathrm{pCDH}$ and PC-3/miR-186 cells were injected into the subcutaneous of 6-week-old male nude mice (weight, $16-18 \mathrm{~g} ; \mathrm{n}=4$ mice/group) and tumor growth was detected. All mice were sacrificed after 28 days using carbon dioxide euthanasia. Tumors were then resected and measured.

Bioinformatics analysis. Candidate target genes for miR-186 were searched for using TargetScan (targetscan.org), miRanda (microrna.org) and miRwalk (zmf.umm.uni-heidelberg.de). The relationship between the candidate target genes and PCa was also investigated using published articles in the PubMed database (ncbi.nlm.nih.gov/pubmed).

Statistical analysis. All the experiments were performed at least three times, and all of the results were expressed as the mean \pm standard deviation. Statistical analysis was performed by Student's t-test using SPSS v. 16.0 software (SPSS, Inc., Chicago, IL, USA). $\mathrm{P}<0.05$ was used to indicate a statistically significant difference.

\section{Results}

Identification of miR-186 as a tumor suppressor in PCa. Previous results have demonstrated that the expression of miR-186 in PCa specimens was downregulated compared with the corresponding non-malignant specimens and benign prostatic hyperplasia (BPH) samples, and that this was inversely correlated with the clinicopathological parameters (11). In order to define the expression of miR-186 in prostate cell lines, miR-186 expression levels were detected in the RWPE-1 cell line and three PCa cell lines (PC-3, DU145 and Lncap). As shown in Fig. 1, the miR-186 expression was significantly decreased in the PCa cell lines in comparison with RWPE-1, and it was the lowest in PC-3, which was selected for further overexpression studies. These results indicated that miR-186 expression is frequently downregulated in PCa cell lines and that this may be involved in the development of PCa.

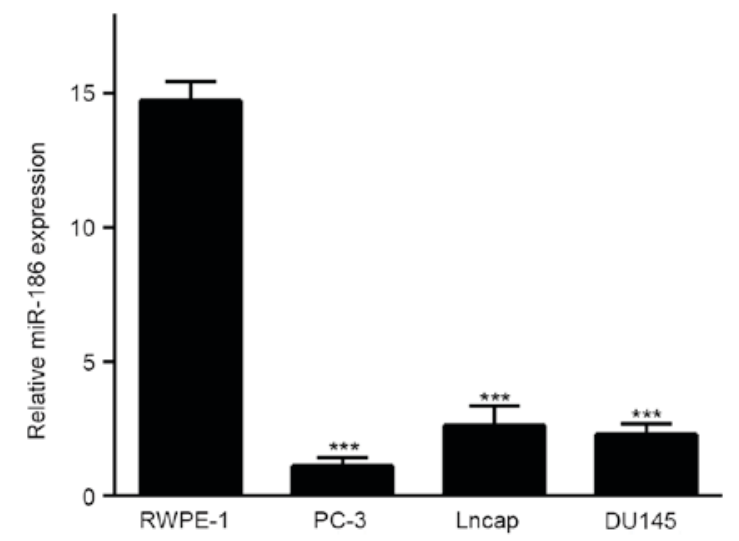

Figure 1. Expression levels of miR-186 in prostate cancer cell lines. The expression of miR-186 in adult human prostatic epithelial cells and human prostate cancer cell lines were analyzed using quantitative polymerase chain reaction. ${ }^{* * *} \mathrm{P}<0.001$ vs. RWPE-1. miRNA, microRNA.

miR-186 inhibits $P C a$ cell growth in vitro. The progression of cancer depends on the malignant proliferation of cancer cells to some degree. To calculate the biological function of miR-186 in PCa, the PCa cell line PC-3 that has the lowest miR-186 expression, was stably infected with a pCDH-miR-186 lentivirus, and $\mathrm{pCDH}$ was used to act as a negative control (Fig. 2A). miR-186 overexpression increased the percentage of cells in the G0/G1 phase and decreased the percentage of cells in the S phase (Fig. 2B). Furthermore, the CCK8 assays implied that the ectopic overexpression of miR-186 suppressed the proliferation of PC-3 cells (Fig. 2C).

miR-186 suppresses the tumor growth of PCa cells in vivo. To test whether miR-186 can suppress tumor growth of $\mathrm{PCa}$ cells in vivo, nude mice were randomly assigned in two groups and received subcutaneous injection of PC-3/miR-186 and PC-3/pCDH cells. In total, 4 weeks after injection, the mice injected with PC-3/miR-186 cells displayed evidently smaller tumors compared with the mice injected with PC-3/pCDH cells (Fig. 3A). Additionally, the mice injected with PC-3/pCDH cells displayed significantly increased weight compared with that of mice injected with PC-3/miR-186 cells (Fig. 3B and C). These data demonstrate that, consistent with the in vitro data of the present study, miR-186 inhibits the tumor growth of $\mathrm{PCa}$ cells in vivo.

miR-186 inhibits YY1 and CDK6 expression by targeting their 3'-UTRs. To determine the potential mechanism by which miR-186 suppresses the tumor growth of PCa cells, four in silico algorithms (Targetscan, miRanda, and miRwalk) were used to forecast the target genes of miR-186 (14), and these potential candidates were obtained, including CDK6, MAP3K2, NCOA2, VEGFA, PMEPA, RBBP9, XIAP, TGFBR2, YY1 and FOLH1. The majority of these candidates are irregularly expressed in PCa compared with normal tissue or related to cell proliferation (15-17). Next, RT-qPCR was used to detect the expression of these target genes in PC-3/pCDH or PC-3/miR-186. The expression of YY1, CDK6, VEGFA and MAP3K2 were found to be decreased $>30 \%$ upon ectopic miR-186 overexpression in PC-3 cells (Fig. 4A). 

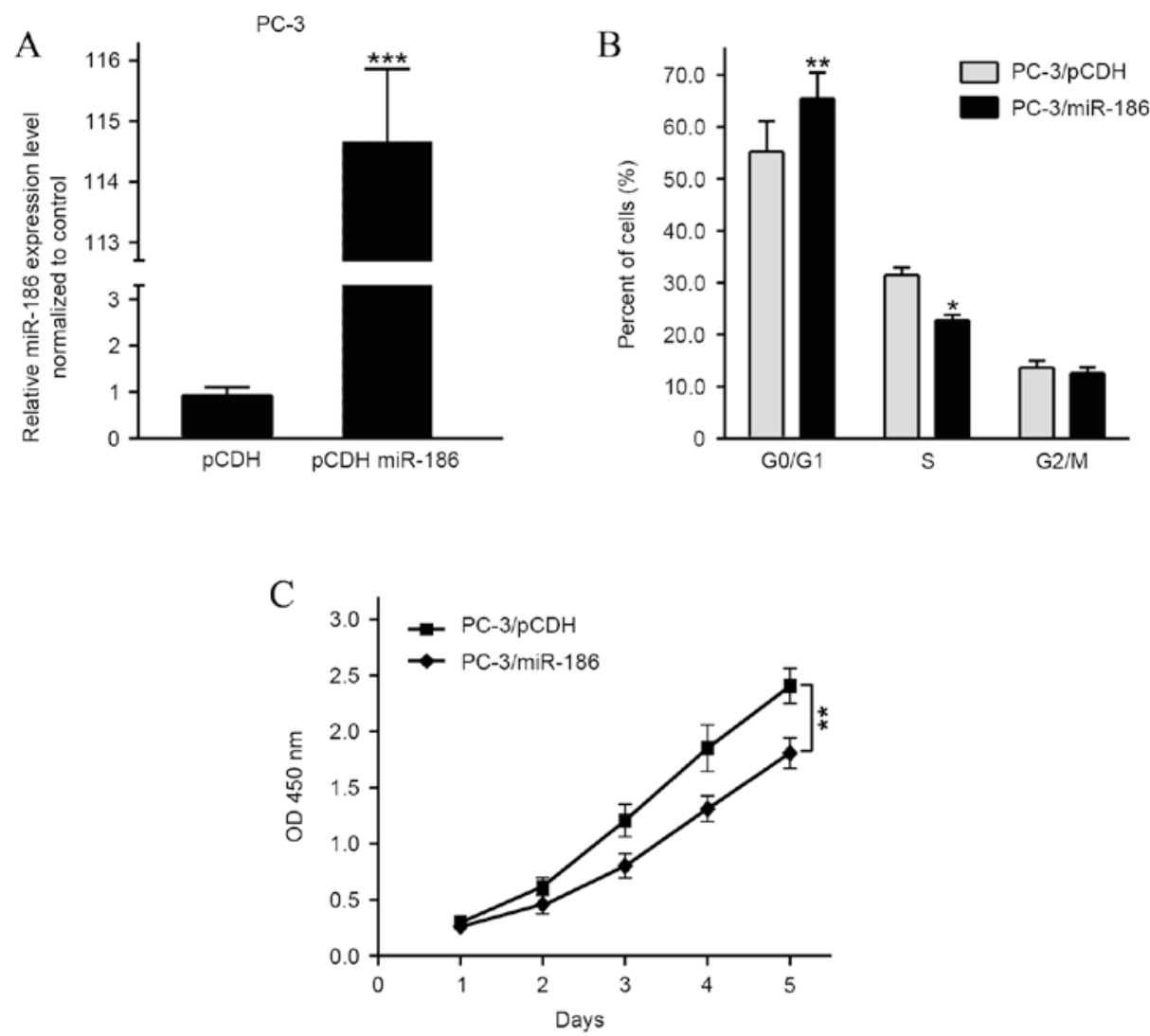

Figure 2. miR-186 inhibits prostate cancer cell proliferation. (A) The miR-186 expression level in PC-3 cells after transfection with pCDH-miR-186 or pCDH were detected using quantitative polymerase chain reaction, where pCDH acted as a negative control. (B) The cell cycle phases of PC-3 cell transfection with pCDH-miR-186 or pCDH were analyzed using flow cytometry, where pCDH acted as a negative control. (C) Effect on cell proliferation of miR-186 overexpression in PC-3 cells was determined by the Cell Counting Kit- 8 assay, where pCDH acted as a negative control. ${ }^{*} \mathrm{P}<0.05,{ }^{* * *} \mathrm{P}<0.01$ and ${ }^{* * * *} \mathrm{P}<0.001$. miRNA, microRNA.

\section{A}

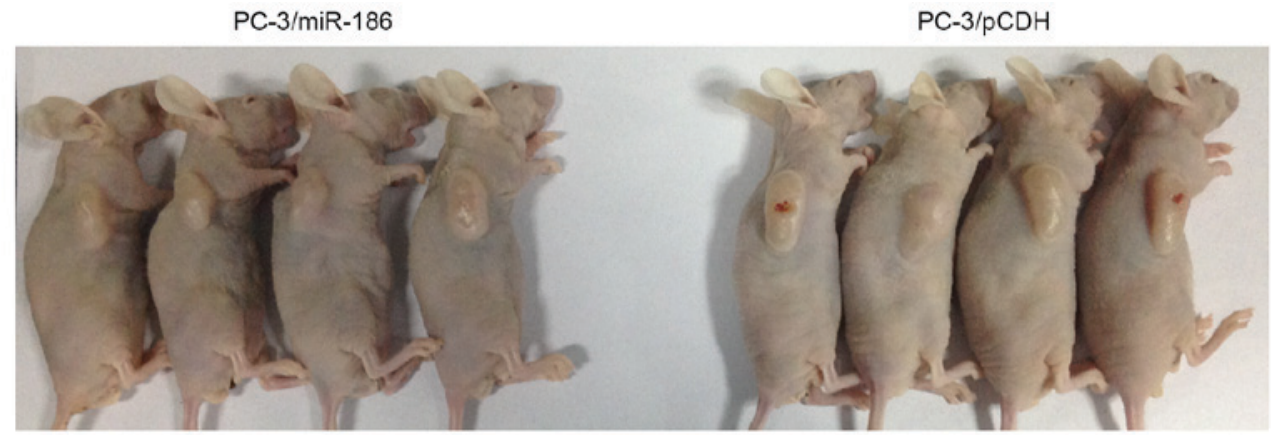

B

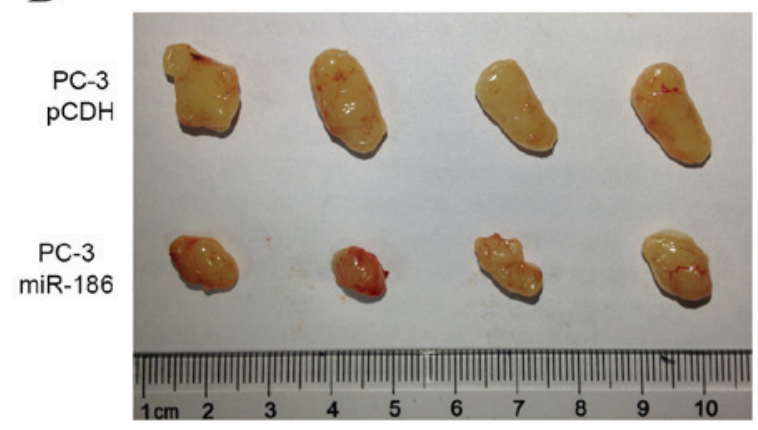

$\mathrm{C}$



Figure 3. miR-186 suppresses tumor growth of prostate cancer cells in vivo. (A) Four weeks after PC-3/pCDH and PC-3/miR-186 cells were injected into the subcutaneous, mice were euthanized and examined for the growth of subcutaneous tumor. (B) Tumor sizes and (C) weights from the mice in the experiment were measured. ${ }^{* * *} \mathrm{P}<0.001$. miRNA, microRNA. 
A

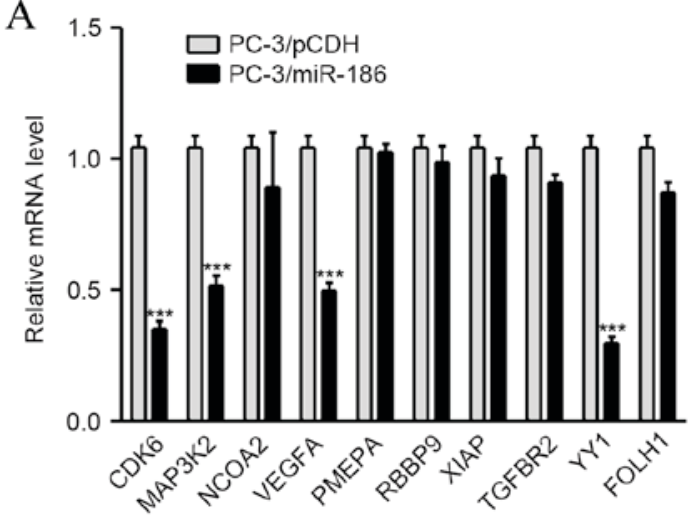

C

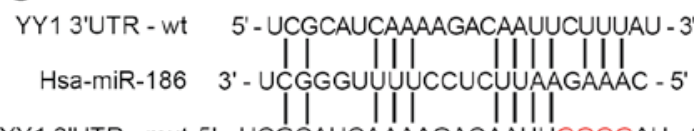

YY1 3'UTR - mut 5' - UCGCAUCAAAAGACAAUUGGGGAU -

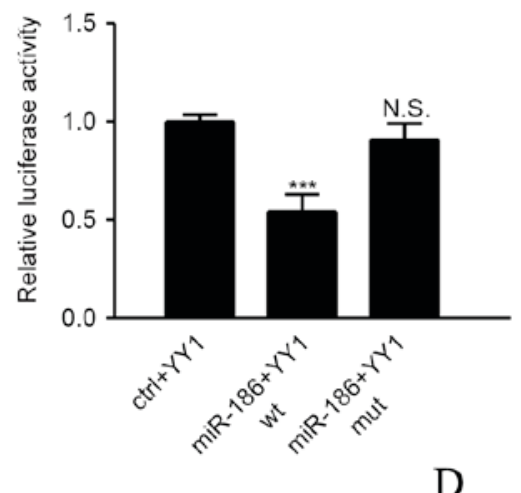

$\mathrm{D}$

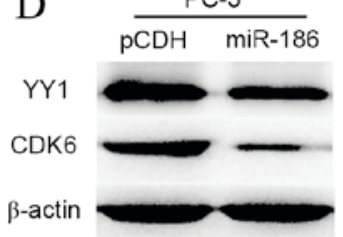

B

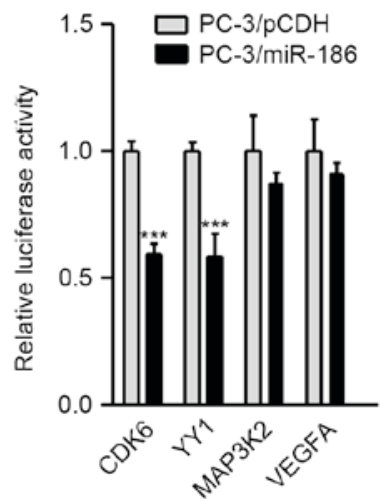

CDK6 3'UTR - wt 5' - UGGAAUUUUUCAgCAUUCUUUAG - 3'



CDK6 3'UTR - mut 5' - UGGAAUUUUUCAGCAUUGGGGAG - 3'

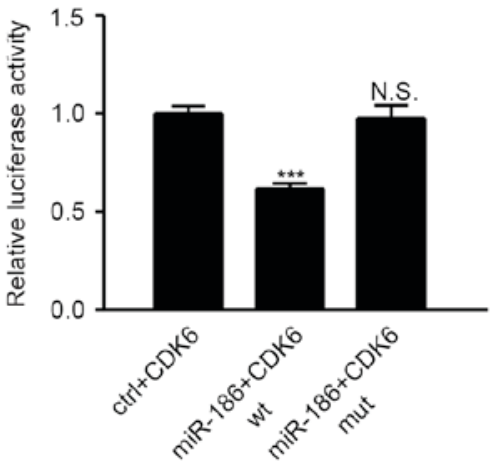

Figure 4. YY1 and CDK6 are direct targets of miR-186. (A) The mRNA levels of predicted target genes of miR-186 in PC-3/miR-186 and PC-3/pCDH cells were detected using quantitative polymerase chain reaction. (B) Effects of miR-186 overexpression on the activity of the 3'-UTRs of target genes in PC-3 cells were analyzed by the dual luciferase reporter assay, where PC-3/pCDH acted as a negative control. (C) Effects of miR-186 expression on the activity of wild-type and mutant 3'-UTRs of YY1 and CDK6 were analyzed by the dual luciferase reporter assay. (D) Effects of miR-186 overexpression in PC-3 cells on the expression of YY1 and CDK6 were detected by Western blotting, where PC-3/pCDH acted as negative controls. ${ }^{*} \mathrm{P}<0.05,{ }^{* *} \mathrm{P}<0.01$ and ${ }^{* * * *} \mathrm{P}<0.001$. N.S, no significance; YY1, Yin Yang 1; CDK6, cyclin-dependent kinase 6; UTRs, untranslated regions; miRNA, microRNA.

Next, the wild type or mutant 3'-UTRs of YY1, CDK6, VEGFA and MAP3K2 were cloned into luciferase constructs to define whether miR-186 can directly bind to these genes. As shown in Fig. 4B, the overexpression of miR-186 conspicuously decreased the luciferase activity of YY1 and CDK6 by 40-45\% compared to the controls. Additionally, these miR-186 binding sites in the 3'-UTRs of YY1 and CDK6 were obliterated by QuickChange PCR (18). However, it was revealed that the mutant binding sites of miR-186 in the target genes YY1 and CDK6 3'-UTRs abolished the miR-186-mediated inhibitory effect on the luciferase activity (Fig. 4C). Western blot analyses were also performed to obtain the expression levels of the YY1 and CDK6 protein. As shown in Fig. 4D, the levels of YY1 and CDK6 were evidently decreased in PC-3/miR-186 cells compared with PC-3/pCDH cells. All of these data imply that YY1 and CDK6 are direct targets of miR-186 in PCa cells.

\section{Discussion}

The aberrant expression of miRNAs has been shown to be important in tumor progression, and these miRNAs inhibit their target genes by binding to the 3'-UTRs of their target genes (8-10). Understanding of the mechanism between miRNAs and their target genes during tumor progression may allow for the identification of several new diagnostic and therapeutic biomarkers. Various miRNAs have been demonstrated to be involved in PCa. For example, miR-631 inhibits the invasion and migration of PCa cells by downregulating ZAP70 expression (19). miR-221 and miR-222 are upregulated in the PCa target aplysia Ras homolog I to regulate the cell cycle and apoptosis (20). Notably, comparative analysis between malignant and non-malignant prostate tissue samples revealed that miR-186 was downregulated in PCa tissues (11). 
Furthermore, the present study identified that miR-186 was downregulated in the PCa cell lines compared with the human prostatic epithelial cell lines. Additionally, miR-186 targets the 3'-UTRs of YY1 and CDK6 mRNAs and downregulates YY1 and CDK6 expression, inhibiting PCa cell proliferation and tumor growth.

YY1 is a member of the Polycomb Group protein family, which was found to function as a transcription factor and is important in biological processes such as development, replication and cell proliferation $(21,22)$. The expression of YY1 was higher in PCa and in prostate intraepithelial neoplasia samples compared to BPH tissue samples (23). Downregulation of YY1 expression in ovarian cancer cell lines can inhibit cell growth and proliferation (24). CDK6, a member of the CDK family, was known to play a critically stimulative role in the cell cycle (25). After inhibiting the activity of CDK6, G1 was arrested and cell proliferation was suppressed (26). Furthermore, a recent study demonstrated that CDK6 overexpression in $\mathrm{PCa}$ is correlated with the development of human PCa (27). These studies demonstrate that YY1 and CDK6 are oncogenes that may contribute to tumor growth and cell proliferation. In the present study, Targetscan, miRanda and miRwalk databases were used in order to identify that there were highly conserved miR-186 binding sites in the 3'-UTRs of YY1 and CDK6, indicating that miR-186 may inhibit PCa cell proliferation and tumor growth by downregulating the expression of YY1 and CDK6, at least to a certain extent.

In conclusion, the results of the present study suggest that miR-186 is a novel tumor suppressor in the progression of $\mathrm{PCa}$. Furthermore, miR-186 expression is inversely associated with the proliferation of PC-3 cells and tumor growth. Furthermore, miR-186 may inhibit PCa cell proliferation and tumor growth by targeting YY1 and CDK6. Thus, the mechanism of miR-186 in PCa will provide new strategies to diagnose and treat PCa.

\section{References}

1. Siegel RL, Miller KD and Jemal A: Cancer statistics, 2015. CA Cancer J Clin 65: 5-29, 2015.

2. Nishikawa R, Goto Y, Sakamoto S, Chiyomaru T, Enokida H, Kojima S, Kinoshita T, Yamamoto N, Nakagawa M, Naya Y, et al: Tumor-suppressive microRNA-218 inhibits cancer cell migration and invasion via targeting of LASP1 in prostate cancer. Cancer Sci 105: 802-811, 2014.

3. Bott SR, Birtle AJ, Taylor CJ and Kirby RS: Prostate cancer management: 2 . An update on locally advanced and metastatic disease. Postgrad Med J 79: 643-645, 2003.

4. Patil PA and Magi-Galluzzi C: MicroRNA in prostate cancer: Practical aspects. Histol Histopathol 30: 1379-1396, 2015.

5. Carthew RW and Sontheimer EJ: Origins and mechanisms of miRNAs and siRNAs. Cell 136: 642-655, 2009.

6. Yates LA, Norbury CJ and Gilbert RJ: The long and short of microRNA. Cell 153: 516-519, 2013.

7. Calin GA and Croce CM: MicroRNA-cancer connection: The beginning of a new tale. Cancer Res 66: 7390-7394, 2006.

8. Cai ZK, Chen Q, Chen YB, Gu M, Zheng DC, Zhou J and Wang Z: microRNA-155 promotes the proliferation of prostate cancer cells by targeting annexin 7. Mol Med Rep 11: 533-538, 2015.
9. Tong SJ, Liu J, Wang X and Qu LX: microRNA-181 promotes prostate cancer cell proliferation by regulating DAX-1 expression. Exp Ther Med 8: 1296-1300, 2014.

10. Nishikawa R, Goto Y, Kojima S, Enokida H, Chiyomaru T, Kinoshita T, Sakamoto S, Fuse M, Nakagawa M, Naya Y, et al: Tumor-suppressive microRNA-29s inhibit cancer cell migration and invasion via targeting LAMC1 in prostate cancer. Int $\mathrm{J}$ Oncol 45: 401-410, 2014.

11. Erdmann K, Kaulke K, Thomae C, Huebner D, Sergon M, Froehner M, Wirth MP and Fuessel S: Elevated expression of prostate cancer-associated genes is linked to down-regulation of microRNAs. BMC Cancer 14: 82, 2014.

12. Fang X, Cai Y, Liu J, Wang Z, Wu Q, Zhang Z, Yang CJ, Yuan L and Ouyang G: Twist 2 contributes to breast cancer progression by promoting an epithelial-mesenchymal transition and cancer stem-like cell self-renewal. Oncogene 30: 4707-4720, 2011.

13. Zhang C, Zhang Y, Ding W, Lin Y, Huang Z and Luo Q: MiR-33a suppresses breast cancer cell proliferation and metastasis by targeting ADAM9 and ROS1. Protein Cell 6: 881-889, 2015.

14. Grimson A, Farh KK, Johnston WK, Garrett-Engele P, Lim LP and Bartel DP: MicroRNA targeting specificity in mammals: Determinants beyond seed pairing. Mol Cell 27: 91-105, 2007.

15. Liu Y, Song H, Pan J and Zhao J: Comprehensive gene expression analysis reveals multiple signal pathways associated with prostate cancer. J Appl Genet 55: 117-124, 2014.

16. Eroglu C, Seçme M, Bağci G and Dodurga Y: Assessment of the anticancer mechanism of ferulic acid via cell cycle and apoptotic pathways in human prostate cancer cell lines. Tumour Biol 36: 9437-9446, 2015.

17. Jones AC, Antillon KS, Jenkins SM, Janos SN, Overton HN, Shoshan DS, Fischer EG, Trujillo KA and Bisoffi M: Prostate field cancerization: Deregulated expression of macrophage inhibitory cytokine 1 (MIC-1) and platelet derived growth factor A (PDGF-A) in tumor adjacent tissue. PLoS One 10: e0119314, 2015.

18. Zheng L, Baumann U and Reymond JL: An efficient one-step site-directed and site-saturation mutagenesis protocol. Nucleic Acids Res 32: e115, 2004.

19. Fu D, Liu B, Zang LE and Jiang H: MiR-631/ZAP70: A novel axis in the migration and invasion of prostate cancer cells. Biochem Biophys Res Commun 469: 345-351, 2016.

20. Chen Y, Zaman MS, Deng G, Majid S, Saini S, Liu J, Tanaka Y and Dahiya R: MicroRNAs 221/222 and genistein-mediated regulation of ARHI tumor suppressor gene in prostate cancer. Cancer Prev Res (Phila) 4: 76-86, 2011.

21. Deng Z, Cao P, Wan MM and Sui G: Yin Yang 1: A multifaceted protein beyond a transcription factor. Transcription 1: 81-84, 2010.

22. Castellano G, Torrisi E, Ligresti G, Malaponte G, Militello L, Russo AE, McCubrey JA, Canevari S and Libra M: The involvement of the transcription factor Yin Yang 1 in cancer development and progression. Cell Cycle 8: 1367-1372, 2009.

23. Seligson D, Horvath S, Huerta-Yepez S, Hanna S, Garban H, Roberts A, Shi T, Liu X, Chia D, Goodglick L and Bonavida B: Expression of transcription factor Yin Yang 1 in prostate cancer. Int J Oncol 27: 131-141, 2005.

24. Matsumura N, Huang Z, Baba T, Lee PS, Barnett JC, Mori S, Chang JT, Kuo WL, Gusberg AH, Whitaker RS, et al: Yin yang 1 modulates taxane response in epithelial ovarian cancer. Mol Cancer Res 7: 210-220, 2009.

25. Sridhar J, Akula N and Pattabiraman N: Selectivity and potency of cyclin-dependent kinase inhibitors. AAPS J 8: E204-E221, 2006.

26. Wang QM, Luo X and Studzinski GP: Cyclin-dependent kinase 6 is the principal target of p27/Kip1 regulation of the G1-phase traverse in 1,25-dihydroxyvitamin D3-treated HL60 cells. Cancer Res 57: 2851-2855, 1997.

27. Lim JT, Mansukhani M and Weinstein IB: Cyclin-dependent kinase 6 associates with the androgen receptor and enhances its transcriptional activity in prostate cancer cells. Proc Natl Acad Sci USA 102: 5156-5161, 2005. 